\title{
A New Perspective to Evaluate Doppler Vascular Impedance in Hypertensive Disorders Complicating Pregnancy: Multilevel Modeling Established in a Case Control Study
}

\author{
Ting Yuan1, Ting Zhang1, Chao Li², Zhen Han ${ }^{*}$ \\ ${ }^{1}$ Department of Obstetrics \& Gynecology, First Affiliated Hospital of Xi'an Jiaotong University College of \\ Medicine, Xi'an, China \\ ${ }^{2}$ Department of Public Health, Xi'an Jiaotong University College of Medicine, Xi'an, China \\ Email: y.t.1109@qq.com, tingting711@stu.xjtu.edu.cn, 240540529@qq.com, ${ }^{*}$ hanamy02@163.com
}

Received 5 May 2015; accepted 20 June 2015; published 23 June 2015

Copyright $@ 2015$ by authors and Scientific Research Publishing Inc.

This work is licensed under the Creative Commons Attribution International License (CC BY).

http://creativecommons.org/licenses/by/4.0/

(c) (i) Open Access

\section{Abstract}

Background: There have been researches on the evaluation of Doppler vascular impedance in hypertensive disorders complicating pregnancy (HDCP). With respect to the method of analysis used and the conclusions drawn in previous studies, different vessels were usually viewed separately and independently. This study was designed to evaluate Doppler vascular impedance changes in HDCP from a new perspective, with original thought and insight into an ordinary issue. Methods: 273 pregnant women (110 hypertensive pregnancies and 163 normotensive pregnancies) were randomly included in a grouping case-control study conducted from February 10, 2011 to April 30, 2013. All women in the study underwent Doppler measurements of six different vessels including the umbilical artery, the uterine arteries, the placental bed spiral artery, the fetal middle cerebral artery and the fetal renal artery. Doppler vascular impedance was presented as pulsatility index (PI), resistance index (RI), and systolic and diastolic ratio (S/D). Doppler changes in the hypertensive and normotensive groups were assessed by the multilevel modeling approach with univariate and multivariate-adjusted analyses. Results: According to multilevel modeling approach with multivariate-adjusted analysis, a relatively average evaluation on Doppler vascular impedance was provided. Hypertension was significantly associated with positive effects on PI, RI and S/D values (coefficients were $0.10,0.03$ and 0.08 , respectively; $95 \%$ CIs were $0.06-0.14,0.02$ 0.04 and $0.04-0.11$, respectively; $P$ values were all less than 0.001 ) in comparison with normo-

${ }^{*}$ Corresponding author.

How to cite this paper: Yuan, T., Zhang, T., Li, C. and Han, Z. (2015) A New Perspective to Evaluate Doppler Vascular Impedance in Hypertensive Disorders Complicating Pregnancy: Multilevel Modeling Established in a Case Control Study. Open Journal of Obstetrics and Gynecology, 5, 350-359. http://dx.doi.org/10.4236/ojog.2015.56051 
tensive group. Conclusion: According to an overall evaluation, Doppler vascular impedance in hypertensive disorders complicating pregnancy was higher than in normotensive pregnancy. The novel thought and approach applied in this research may bring about inspirations for better understanding and assessment of the disease.

\section{Keywords}

\section{Pregnancy, Doppler, Vascular Impedance, Multilevel Modeling}

\section{Background}

Hypertensive disorders complicating pregnancy (HDCP) denotes a group of common medical complications that may occur in pregnancy and that are a major cause of maternal and neonatal mortality and morbidity [1]. The prevalence of HDCP is approximately $5.22 \%$ and $8 \%-10 \%$ of all pregnancies in China and worldwide respectively [2] [3]. The disease, the clinical manifestations of which include hypertension and proteinuria, can occur during pregnancy or delivery. Hypertensive disorders complicating pregnancy may result in severe maternal and fetal complications including cardiovascular and cerebrovascular disease, liver and kidney dysfunction, HELLP syndrome, placental abruption and in fetal growth restriction, fetal death, stillbirth, preterm birth and neonatal asphyxia [1]. Although recent decades have brought important advances in our understanding of the etiology and pathophysiological mechanisms of HDCP [4], most of the factors leading to the disease still remain unclear.

Doppler measurement is considered to be one of the most important conventional techniques for assessing antenatal conditions, due to its wide availability, its clinical applicability and its non-invasiveness [5]. Doppler evaluation of vascular impedance in hypertensive pregnancy has been a focal point in recent years. Blood flow indices obtained using this technique can provide indirect evidence regarding maternal and fetal hemodynamics and circulatory changes; based on extensive research that has led to deep understanding of Doppler measurement, appropriate clinical management can then be carried out promptly. The currently available literature includes reports of numerous studies on Doppler vascular impedance in hypertensive pregnancy [6]-[13]. Most of these studies investigated Doppler vascular impedance based on one or several vessels, such as the umbilical artery, the uterine artery, and the fetal middle cerebral artery. However, with respect to the method of analysis used and the conclusions drawn in these studies, different vessels were usually viewed separately and independently.

In the present work, we adopted a different approach and used a novel method of analysis to investigate vascular impedance in HDCP by Doppler measurements. From a different aspect, this research may contribute to deep understanding of the disease.

\section{Methods}

The study was conducted at the First Affiliated Hospital of Xi'an Jiaotong University, Shaanxi, China, from February 10, 2011 to April 30, 2013. It was a case-control study in which we randomly included 110 women with HDCP and 163 normotensive pregnancies as controls. All of the women were at or beyond 26 weeks' gestation. Gestational age was calculated by LMP or confirmed based on the results of first trimester ultrasound. The cases were consisted of 11 gestational hypertension, 88 preeclampsia, 1 eclampsia, 8 superimposed preeclampsia, and 2 chronic hypertension.

The criteria used for the diagnosis of HDCP were those listed in Williams Obstetrics (23 ${ }^{\text {rd }}$ edition) and included: 1) gestational hypertension (hypertension for the first time during pregnancy without proteinuria); 2) preeclampsia (hypertension after 20 weeks' gestation with proteinuria); 3) eclampsia (seizures following preeclampsia); 4) preeclampsia superimposed on chronic hypertension (new-onset proteinuria in hypertensive women but no proteinuria before 20 weeks' gestation or a sudden increase in proteinuria or blood pressure or platelet count $<100,000 / \mu \mathrm{L}$ in women with hypertension and proteinuria before 20 weeks' gestation); 5) chronic hypertension in pregnancy (hypertension before pregnancy or hypertension diagnosed before 20 weeks' gestation not attributable to gestational trophoblastic disease or hypertension first diagnosed after 20 weeks' gestation and persisting longer than 12 weeks postpartum) [14]. Hypertension was defined as systolic blood pressure $\geq 140$ $\mathrm{mmHg}$ and/or diastolic blood pressure $\geq 90 \mathrm{mmHg}$ on at least two occasions at a 4-hour interval. Proteinuria 
was defined as $\geq 300 \mathrm{mg}$ protein/24 hours or $\geq 1+$ dipstick.

Women with fetal chromosomal abnormalities, fetal malformations, placental or umbilical anomalies, associated complications of pregnancy (diabetes mellitus, intrahepatic cholestasis, thrombophilia, and thrombopenia, among others), systemic vascular or autoimmune disorders, multiple pregnancies, rupture of the amniotic membrane and women receiving vasoactive drugs or in active labor were excluded from the study. The control participants were normotensive.

The study was approved by the ethical and research committee of the hospital. The purpose and procedures of the study were explained to all enrolled participants, and a written informed consent form was obtained from each participant.

Routine ultrasound and Doppler flow spectrum measurements of the umbilical artery, the uterine arteries, the fetal middle cerebral artery, the fetal renal artery and the placental spiral artery were carried out in all cases. All ultrasound scans were performed by a single investigator (Ting Yuan) using a Voluson E8 (GE Medical Systems, Milwaukee, WI, USA) ultrasound system. The investigator was blinded to cases and controls.

The umbilical artery Doppler flow spectrum was recorded from a free floating portion of the umbilical cord during minimal fetal activity and the absence of fetal breathing (Figure 1). The fetal middle cerebral artery Doppler flow spectrum was recorded in a transverse view of the fetal brain, with the Doppler gate placed on the vessel approximately $1 \mathrm{~cm}$ distal to the circle of Willis (Figure 2). The fetal renal artery flow spectrum was performed in a bilateral coronal section of the fetal kidneys with the Doppler gate placed on the trunk of the renal artery near the hilus (Figure 3). Uterine artery Doppler was carried out by identifying the vessel in an oblique scan with the sample volume distal to the crossing of the uterine artery and the bilateral external iliac artery (Figure 4). The placental spiral artery waveform was obtained with the Doppler gate placed on the area between the placenta and the uterine muscular layer. In all of these measurements, the angle between the ultrasonographic beam and the direction of blood flow was between 15 and 30 degrees (Figure 5). Angle correction was applied and the signal was updated until 3 - 5 similar consecutive waveforms were obtained. The obtained flow indices were presented as pulsatility index (PI), resistance index (RI), and systolic and diastolic ratio(S/D). FGR was defined when the fetus was below the tenth percentile on the curve of weight for gestational age according to ACOG [15].

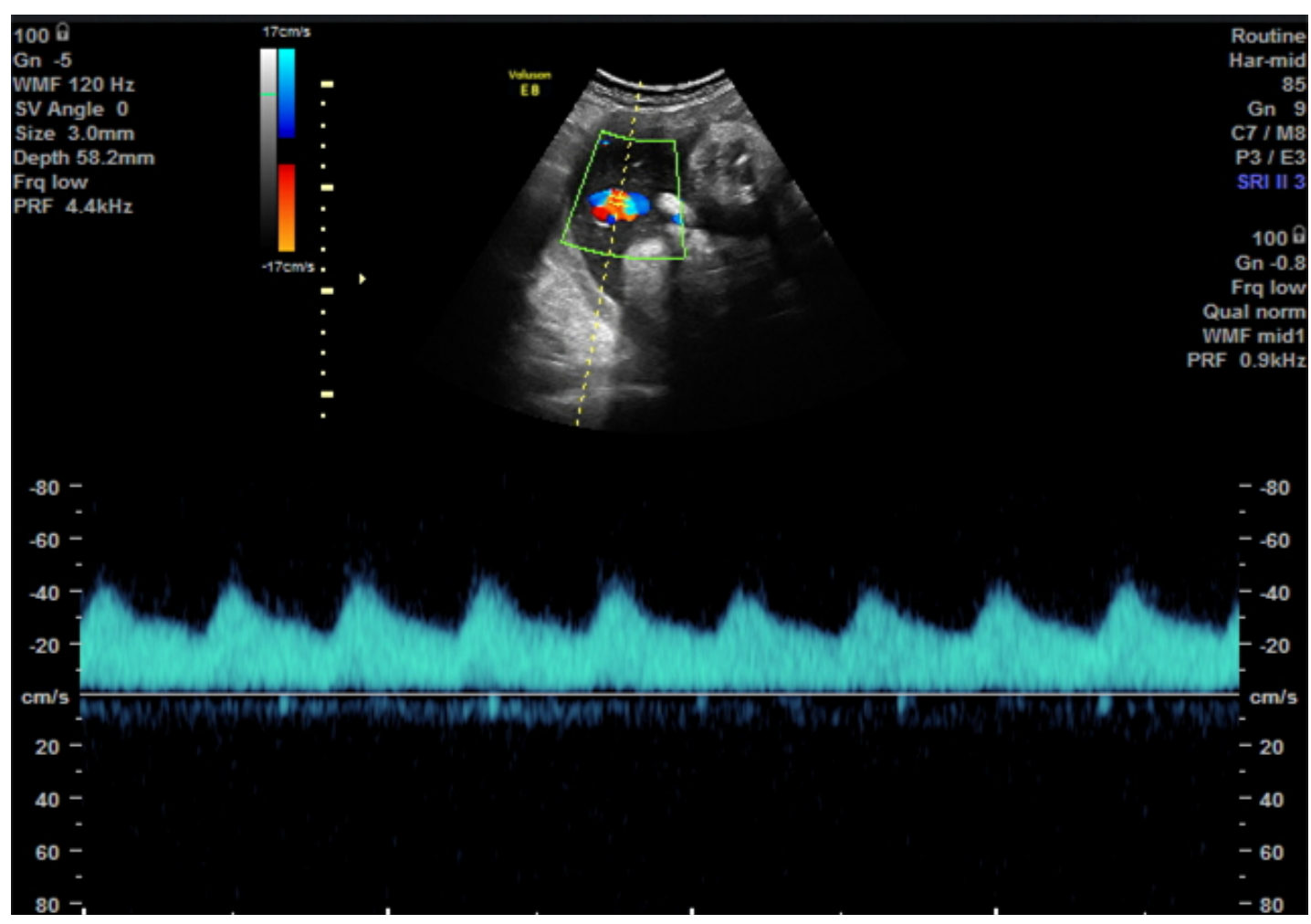

Figure 1. Doppler waveform of umbilical artery (38 weeks’ gestation). 


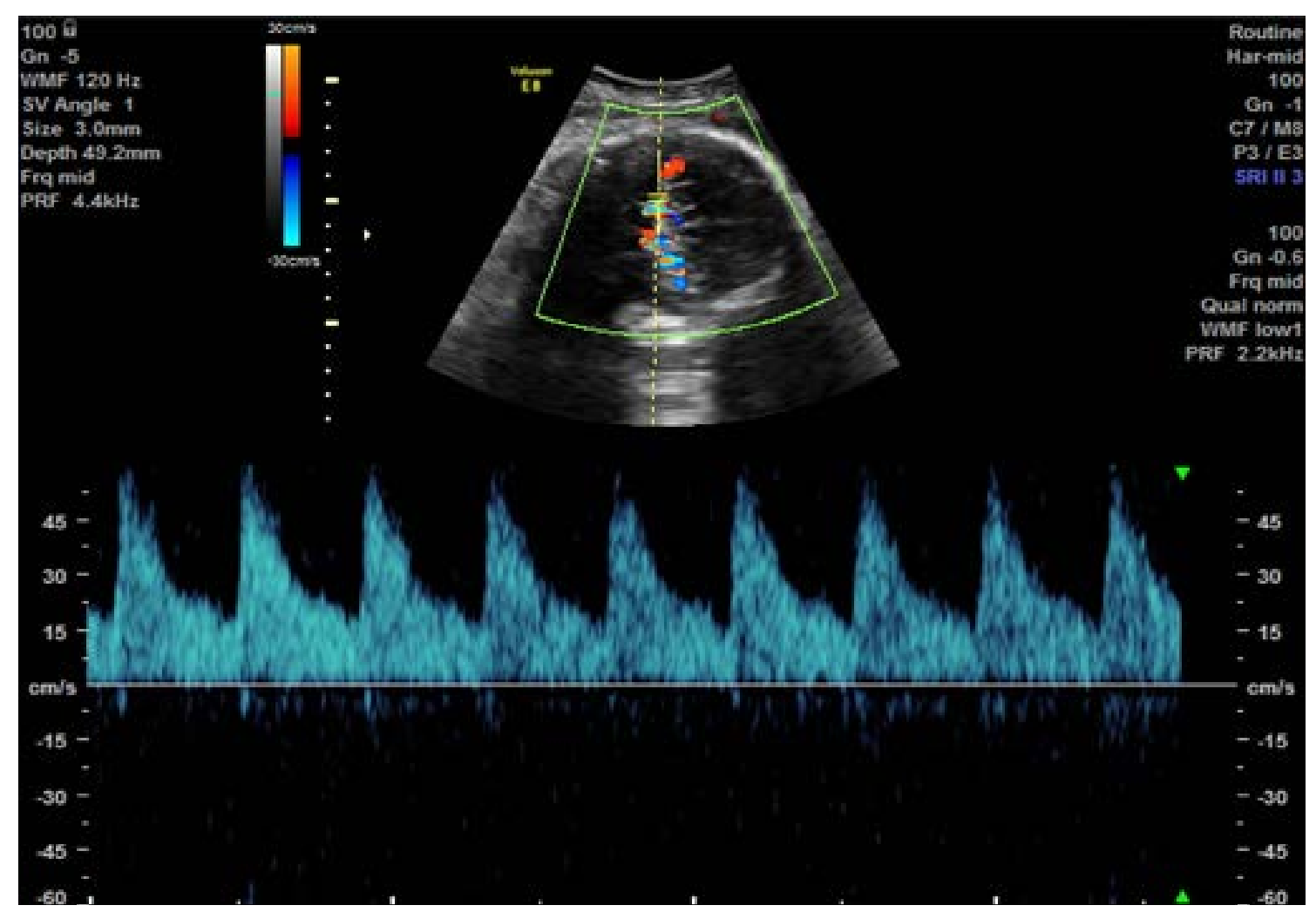

Figure 2. Doppler waveform of the fetal middle cerebral artery (38 weeks' gestation).

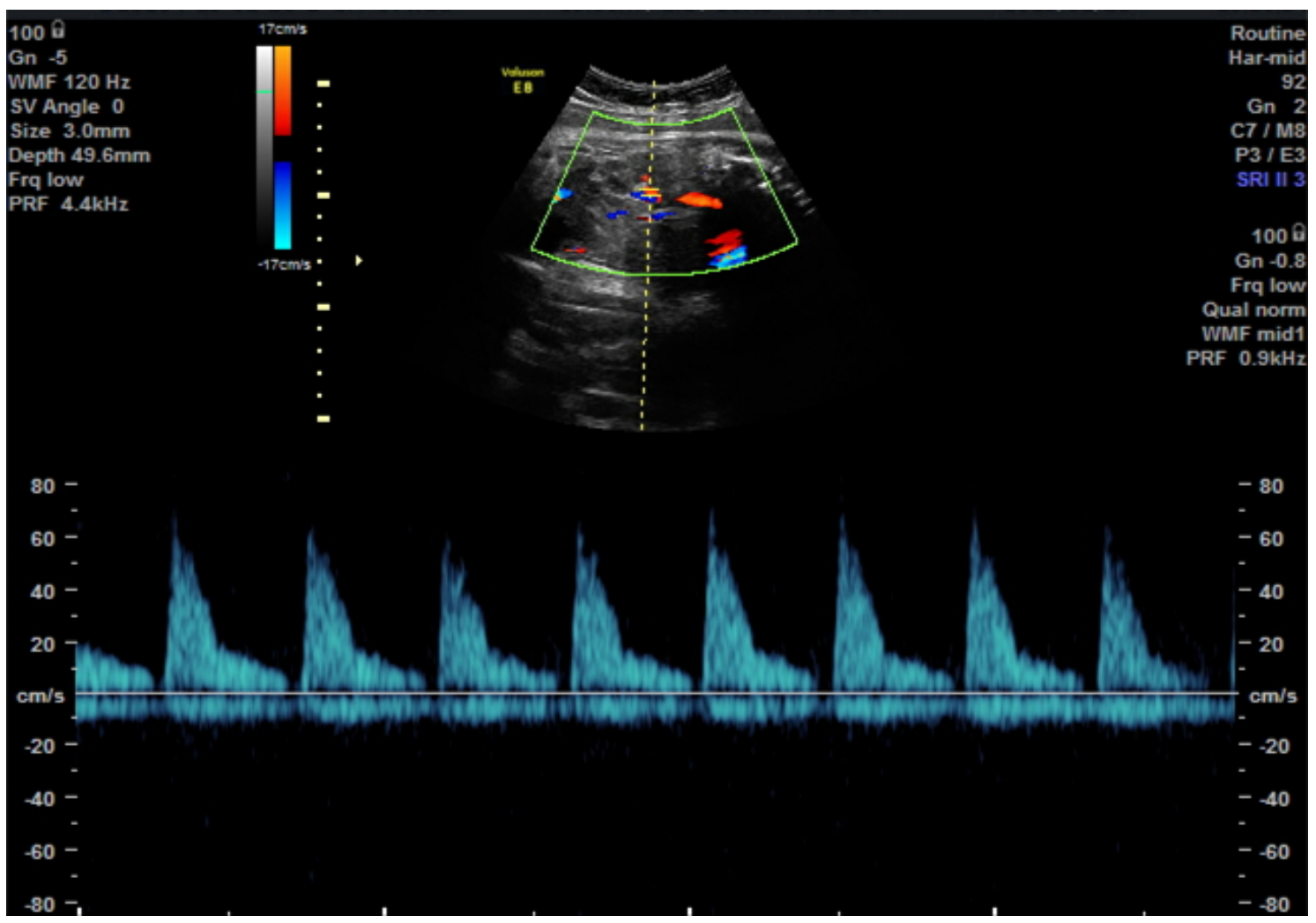

Figure 3. Doppler waveform of the fetal renal artery (38 weeks' gestation).

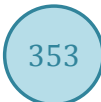






Figure 4. Doppler waveform of the uterine artery (38 weeks’ gestation).

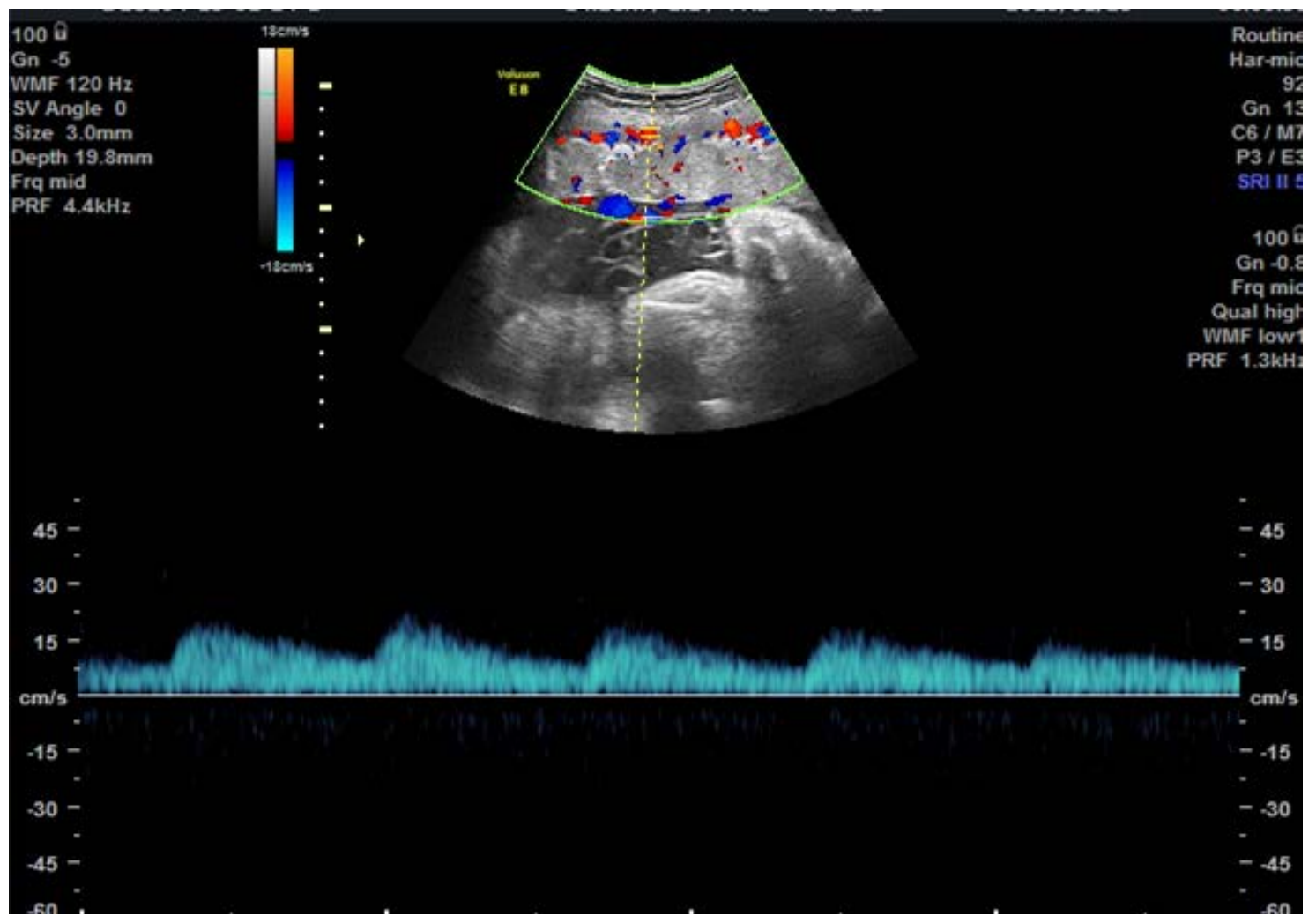

Figure 5. Doppler waveform of the placental bed spiral artery (39 weeks' gestation).

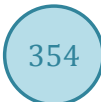


The data on the enrolled pregnant women were checked and double-entered into the data management system. The distribution and pregnancy status of the hypertensive and normotensive groups were presented as Mean with standard deviation or Median (range) for quantitative data and as percentages for categorical variables. Due to the hierarchical structure of the data, a multilevel modeling approach with individual to level 2 and sampling location to level 1 was developed to assess differences in the Doppler indices of the hypertensive and normotensive groups. Estimations of the coefficients and 95\% confidence intervals (CIs) were made according to the case and control groups. Due to the rigorous random sample design used in this study, the baseline information was well balanced between the two groups. In addition, because maternal age, gestational age, parity, FGR, place of residence have been considered to be possibly associated with vascular impedance [16]-[20], we ran a model to assess differences in the Doppler indices between hypertensive and normotensive groups after adjustment for these variables. All $\mathrm{P}$ values were 2 -tailed, and values of $<0.05$ were considered to be statistically significant.

The statistical analysis was performed using STATA version 12.0 (StataCorp, College Station, Texas, USA).

\section{Results}

The demographic and clinical characteristics of the 273 women, including maternal age, gestational age at Doppler examination, nulliparity, place of residence and blood pressure were presented in Table 1.

Table 2 showed there were significant differences between normotensive and hypertensive group in PI, RI and S/D of umbilical artery and middle cerebral artery, impedances of umbilical artery were higher in hypertensive group ( $\mathrm{P}$ values were all less than 0.001), but its impedance of middle cerebral artery were lower ( $\mathrm{P}$ values were $<0.001,0.038,<0.001$ respectively). S/D of fetal renal artery was significantly higher in hypertensive group ( $\mathrm{P}$ value $<0.001$ ). Table 3 showed significant increases in the impedances of maternal vessels in hypertensive group (P values were all less than 0.001).

Table 2 and Table 3 showed that there were discrepancies in the Doppler flow indices values obtained in fetal vessels and maternal vessels between the hypertensive and normotensive groups. The data indicated that Doppler impedances of different locations were different and the discrepancy should be taken into consideration in the subsequent analyses.

Based on the results of univariate analysis, hypertension was associated with a positive effect on PI, RI and S/D values, increasing the mean PI, RI and S/D values by $0.09,0.03$ and 0.07 , respectively (the $95 \%$ CIs were $0.05-0.13,0.02-0.05$ and $0.03-0.11$, respectively) compared with the normotensive group. There were significant differences between the two groups for three indices, and the $\mathrm{P}$ values for these differences were all less than 0.001 . The multivariate-adjusted results revealed similar differences when we included the potential confounders in the analysis. The mean PI, RI and S/D values in the hypertensive group increased by $0.10,0.03$ and 0.08 , respectively (the $95 \%$ CIs were $0.06-0.14,0.02-0.04$ and $0.04-0.11$, respectively) in comparison with the normotensive group. Significant differences still existed and the P values were all less than 0.001 . The results also indicated that gestational age had a significant influence on PI, RI and S/D values in the confounders (Table 4).

\begin{tabular}{|c|c|c|c|}
\hline Characteristic & Hypertensive & Normotensive & $P$ value \\
\hline Number & 110 & 163 & - \\
\hline Maternal age, ys, mean \pm SD & $29.81 \pm 4.86$ & $28.14 \pm 3.72$ & 0.532 \\
\hline Gestational age, wks, mean \pm SD & $35.10 \pm 3.79$ & $34.75 \pm 4.59$ & 0.257 \\
\hline Nulliparity, n, \% & & & 0.075 \\
\hline Yes & $92(83.64)$ & $148(90.80)$ & \\
\hline No & $18(16.36)$ & $15(9.20)$ & \\
\hline Place of residence, n, \% & & & 0.004 \\
\hline Rural & 65 (59.09) & $67(41.10)$ & \\
\hline Urban & $45(40.91)$ & $96(58.90)$ & \\
\hline SBP, mmHg, median (range) & $150(145-180)$ & $115(105-120)$ & $<0.001$ \\
\hline DBP, mmHg, median (range) & $100(90-120)$ & $70(65-80)$ & $<0.001$ \\
\hline
\end{tabular}

SBP, systolic blood pressure; DBP, diastolic blood pressure. 
Table 2. Comparisons of Doppler indices of fetal vessels between the hypertensive and normotensive groups ${ }^{\mathrm{a}}$.

\begin{tabular}{cccc}
\hline Index/group & UmA & MCA & RA \\
PI & & & $1.79 \pm 0.43$ \\
Normotensive & $0.85(0.56-1.72)$ & $1.32(0.65-2.59)$ & $1.78 \pm 0.40$ \\
Hypertensive & $0.89(0.42-1.61)$ & $1.23(0.60-6.95)$ & 0.235 \\
P value & $<0.001$ & $<0.001$ & $0.82 \pm 0.09$ \\
RI & & & $0.83 \pm 0.10$ \\
Normotensive & $0.57(0.42-0.88)$ & $0.73(0.08-0.99)$ & 0.562 \\
Hypertensive & $0.60(0.56-1.03)$ & $0.71(0.45-1.04)$ & $5.45(2.50-40.75)$ \\
P value & $<0.001$ & 0.038 & $5.86(2.52-85.28)$ \\
S/D & & $3.75(1.86-14.03)$ & $<0.001$ \\
Normotensive & $2.34(1.72-6.38)$ & $3.40(1.82-28.44)$ & $<0.001$ \\
Hypertensive & $2.50(1.54-6.92)$ & $<0.001$ & \\
P value &
\end{tabular}

${ }^{\mathrm{a}}$ Index values were presented as the mean \pm SD or median (range); PI, pulsatility index; RI, resistance index; S/D, systolic and diastolic ratio; UmA, umbilical artery; MCA, fetal middle cerebral artery; RA, fetal renal artery.

Table 3. Comparisons of Doppler indices of maternal vessels between the hypertensive and normotensive groups ${ }^{\mathrm{a}}$.

\begin{tabular}{cccc}
\hline Index/group & PSA & lUtA & rUtA \\
\hline PI & & & $0.68(0.01-3.12)$ \\
Normotensive & $0.34(0.13-1.72)$ & $0.64(0.03-3.15)$ & $0.81(0.38-2.88)$ \\
Hypertensive & $0.41(0.19-0.98)$ & $0.88(0.18-2.63)$ & $<0.001$ \\
P value & $<0.001$ & $<0.001$ & $0.46(0.01-0.99)$ \\
RI & & & $0.53(0.30-0.99)$ \\
Normotensive & $0.29(0.12-1.21)$ & $0.45(0.02-0.98)$ & $<0.001$ \\
Hypertensive & $0.35(0.10-1.22)$ & $0.56(0.08-0.99)$ & $1.86(1.01-8.87)$ \\
P value & $<0.001$ & $<0.001$ & $2.11(1.43-8.20)$ \\
S/D & $1.41(1.14-4.11)$ & $1.81(1.02-7.92)$ & $<0.001$ \\
Normotensive & $1.51(1.21-2.68)$ & $2.17(1.12-6.39)$ & $<0.001$ \\
Hypertensive & $<0.001$ & $<$ & \\
P value &
\end{tabular}

${ }^{\mathrm{a}}$ Index values were presented as the mean \pm SD or median (range); PI, pulsatility index; RI, resistance index; S/D, systolic and diastolic ratio; PSA, placental bed spiral artery; lUtA, left uterine artery; rUtA, right uterine artery.

Table 4. Comparisons of Doppler index values of fetal and maternal vessels in multilevel modeling between the hypertensive and normotensive groups ${ }^{\mathrm{a}}$.

\begin{tabular}{|c|c|c|c|c|c|c|}
\hline \multirow{2}{*}{ Index/group } & \multicolumn{3}{|c|}{ Model $1^{\text {b }}$} & \multicolumn{3}{|c|}{ Model $2^{c}$} \\
\hline & Coeff. & $P$ value & $95 \%$ CI & Coeff. & $P$ value & $95 \%$ CI \\
\hline \multicolumn{7}{|l|}{ PI } \\
\hline Normotensive group & 0 & & & 0 & & \\
\hline Hypertensive group & 0.09 & $<0.001$ & $0.05-0.13$ & 0.10 & $<0.001$ & $0.06-0.14$ \\
\hline \multicolumn{7}{|l|}{ RI } \\
\hline Normotensive group & 0 & & & 0 & & \\
\hline Hypertensive group & 0.03 & $<0.001$ & $0.02-0.05$ & 0.03 & $<0.001$ & $0.02-0.04$ \\
\hline \multicolumn{7}{|l|}{$\mathrm{S} / \mathrm{D}$} \\
\hline Normotensive group & 0 & & & 0 & & \\
\hline Hypertensive group & 0.07 & $<0.001$ & $0.03-0.11$ & 0.08 & $<0.001$ & $0.04-0.11$ \\
\hline
\end{tabular}

${ }^{\mathrm{a}}$ Multilevel modeling was used to compare the differences between the values for the hypertensive and normotensive groups, with individual to level 2 and sampling location to level 1 . ${ }^{b}$ Model 1 was univariate. 'Model 2 was adjusted for potential confounders including maternal age, gestational age, nulliparity and place of residence.Gestational age showed a significant influence on PI, RI and S/D ratio. 


\section{Discussion}

Multilevel analysis conducted with potential confounders taken into consideration showed that hypertension had a positive influence on vascular impedance in pregnancy based on comparison between the hypertensive and the normotensive groups.

In most of the studies similar to the current one, researchers compared the Doppler vascular indices of hypertensive and normotensive subjects by investigating one vessel or by investigating several vessels separately. Hypertensive pregnancy is actually a disease associated with placental circulatory dysfunction that gradually results in chronic fetal hypoxia. Progressively increased resistance in the umbilical artery and the fetal renal artery are fetal adjustments for hypoxia and deprivation [6] [7]. At the same time, flow impedance in the fetal middle cerebral artery declines; this is called the "brain sparing effect". This process was identified clinically by the observation of reduced Doppler impedance in the middle cerebral artery [8]-[10]. In addition, the placental bed spiral arteries are the terminal branches of the uterine artery, which are subject to similar histopathological mechanisms and, like the uterine artery, show consistent significance with respect to the effects of hypertension. Early evidence has demonstrated that placentas harvested from hypertensive pregnancies, especially those from preeclamptic pregnancies, often show poor remodeling of the spiral arteries [11], and defective conversion of the arteries has been shown to be associated with increased uterine flow resistance [12] [13].

In the studies described above, the Doppler indices of different arteries were analyzed separately. In contrast, the current study proposes a novel approach to the evaluation of vascular impedance in hypertensive pregnancies. The unique characteristic of the data in our study was that it was obtained from multiple sampling locations within each individual. The sampling locations were selected based on the following principles. First, Doppler measurements of the umbilical artery, the fetal middle cerebral artery, the fetal renal artery, the uterine artery and the placental bed spiral artery are widely used to assess both normal and complicated pregnancies; the procedures used to obtain these Doppler measurements are undisputed and uncomplicated, and the reliability and clinical applicability of Doppler PI of the umbilical artery, fetal middle cerebral artery, fetal renal artery, and uterine artery has been confirmed [21]-[24]. Secondly, pregnant women possess two intrinsic circulations, the maternal or uteroplacental circulation and the fetal or fetoplacental circulation. In assessing vascular conditions during pregnancy, vessels that have the capacity to reflect both circulations should be taken into consideration. Doppler flow changes in the umbilical artery, the fetal middle cerebral artery, and the fetal renal artery are viewed as indirect reflections of the fetoplacental and fetal circulations. Furthermore, the uterine artery and the placental bed spiral artery are essential vessels for assessing uteroplacental circulation.

When Doppler indices obtained at different locations were analyzed separately, impedance increased in some locations (the umbilical artery, the fetal renal artery, the uterine artery and the placental bed spiral artery), whereas it decreased in the fetal middle cerebral artery. However, considered from the point of view of pathophysiology, the reality is that these vascular impedance changes are all placental-based alterations and that hypertensive pregnancy is actually a chronic form of placenta insufficiency disease [6]. When Doppler measurements obtained at different locations were integrated within an individual (mother and fetus), we concluded that vascular impedance was significantly higher in hypertensive pregnant women than in their normotensive counterparts. This study thus provided new insight into the influence of hypertension on the vascular impedance of pregnant women and brought about hints on the placental-based etiology and pathogenesis of hypertensive pregnancy from the aspect of Doppler measurement.

Whereas, Doppler measurement of a single vessel may lead to incomplete assessment and management of some clinical conditions, the use of Doppler measurements of different vessels may contribute to more comprehensive and reliable solutions to some problems. Presumably, the changes, degree of changes and durations of changes of the impedances in different vessels were different. However, our analysis of different locations within the fetal and maternal circulations provided an overall evaluation of the changes in vascular impedance during pregnancy and showed that the vascular impedance indeed increased.

The strength of this study was the application of original thought and insight to an ordinary issue. However, this study also possesses certain limitations. First, we did not include confounding variables such as maternal BMI, socioeconomic conditions, or smoking in our analysis. This was primarily due to the very small number of smokers among pregnant women and especially to the very low level of cigarette use by the pregnant women in the study population. Second, further studies involving a larger number of pregnancies and more diverse sampling locations are necessary to confirm our results. 


\section{Conclusion}

According to an overall evaluation, vascular impedance in hypertensive disorders complicating pregnancy was significantly higher compared with normotensive pregnancy. The multilevel model used in this research may contribute to better understanding and assessment of the disease.

\section{Acknowledgements}

This research was supported by the Scientific Research Foundation for Chinese Overseas Scholars, Education Ministry, China) (No. 10 fund 24).

\section{Competing Interests}

The authors declare that they have no competing interest.

\section{Authors' Contributions}

TY collected the data and prepared the manuscript, TZ undertook quality control process, CL participated in the study design and provided the statistical analysis, ZH conceived of the research and undertook the image scanning.

All authors have read and approved the final manuscript.

\section{Authors' Information}

TY, TZ and CL are all PhD candidates. ZH is $\mathrm{PhD}$, professor and doctoral supervisor.

\section{References}

[1] Duley, L. (2009) The Global Impact of Pre-Eclampsia and Eclampsia. Seminars in Perinatology, 33, 130-137. http://dx.doi.org/10.1053/j.semperi.2009.02.010

[2] Ye, C., Ruan, Y., Zou, L., Li, G., Li, C., Chen, Y., et al. (2014) The 2011 Survey on Hypertensive Disorders of Pregnancy (HDP) in China: Prevalence, Risk Factors, Complications, Pregnancy and Perinatal Outcomes. PLoS ONE, 9, e100180. http://dx.doi.org/10.1371/journal.pone.0100180

[3] Ghulmiyyah, L. and Sibai, B. (2012) Maternal Mortality from Preeclampsia/Eclampsia. Seminars in Perinatology, 36, 56-59. http://dx.doi.org/10.1053/j.semperi.2011.09.011

[4] Jim, B., Sharma, S., Kebede, T. and Acharya, A. (2010) Hypertension in Pregnancy: A Comprehensive Update. Cardiology in Review, 18, 178-189. http://dx.doi.org/10.1097/CRD.0b013e3181c60ca6

[5] Papp, Z. and Fekete, T. (2003) The Evolving Role of Ultrasound in Obstetrics/Gynecology Practice. International Journal of Gynecology Obstetrics, 82, 339-346. http://dx.doi.org/10.1016/S0020-7292(03)00224-8

[6] Dikshit, S. (2011) Fresh Look at the Doppler Changes in Pregnancies with Placental-Based Complications. Journal of Postgraduate Medicine, 57, 138-140. http://dx.doi.org/10.4103/0022-3859.81880

[7] Campbell, S., Vyas, S. and Nicolaides, K.H. (1991) Doppler Investigation of the Fetal Circulation. Journal of Perinatal Medicine, 19, 21-26. http://dx.doi.org/10.1515/jpme.1991.19.1-2.21

[8] Moran, M. and McAuliffe, F.M. (2011) Imaging and Assessment of Placental Function. Journal of Clinical Ultrasound, 39, 390-398. http://dx.doi.org/10.1002/jcu.20846

[9] Baschat, A.A., Gembruch, U., Reiss, I., Gortner, L., Weiner, C.P. and Harman, C.R. (2000) Relationship between Arterial and Venous Doppler and Perinatal Outcome in Fetal Growth Restriction. Ultrasound in Obstetrics Gynecology, 16, 407-413. http://dx.doi.org/10.1046/j.1469-0705.2000.00284.X

[10] Fardiazar, Z., Atashkhouei, S., Yosefzad, Y., Goldust, M. and Torab, R. (2013) Comparison of Fetal Middle Cerebral Arteries, Umbilical and Uterin Artery Color Doppler Ultrasound with Blood Gas Analysis in Pregnancy Complicated by IUGR. Iranian Journal of Reproductive Medicine, 11, 47-51.

[11] Brosens, I.A., Robertson, W.B. and Dixon, H.G. (1972) The Role of the Spiral Arteries in the Pathogenesis of Preeclampsia. Obstetrics Gynecology Annual, 1, 177-191.

[12] Prefumo, F., Sebire, N.J. and Thilaganathan, B. (2004) Decreased Endovascular Trophoblast Invasion in First Trimester Pregnancies with High-Resistance Uterine Artery Doppler Indices. Human Reproduction, 19, 206-209. http://dx.doi.org/10.1093/humrep/deh037 
[13] Olofsson, P., Laurini, R.N. and Marsal, K. (1993) A High Uterine Artery Pulsatility Index Reflects a Defective Development of Placental Bed Spiral Arteries in Pregnancies Complicated by Hypertension and Fetal Growth Retardation. European Journal of Obstetrics \& Gynecology and Reproductive Biology, 49, 161-168. http://dx.doi.org/10.1016/0028-2243(93)90265-E

[14] Cunningham, F.G., Leveno, K.J., Bloom, S.L., Hauth, J.C., Rouse, D.J. and Spong, C.Y. (2010) Pregnancy Hypertension. In: Cunningham, F.G., Leveno, K.J., Bloom, S.L., Hauth, J.C., Rouse, D.J. and Spong, C.Y., Eds., Williams Obstetrics, 23rd Edition, McGraw-Hill, New York, 706-756.

[15] American College of Obstetricians and Gynecologists (2013) ACOG Practice Bulletin No. 134: Fetal Growth Restriction. Obstetrics \& Gynecology, 121, 1122-1133. http://dx.doi.org/10.1097/01.AOG.0000429658.85846.f9

[16] Ertan, A.K., Hendrik, H.J., Tanriverdi, H.A., Bechtold, M. and Schmidt, W. (2003) Fetomaternal Doppler Sonography Nomograms. Clinical and Experimental Obstetrics and Gynecology, 30, 211-216.

[17] Prefumo, F., Bhide, A., Sairam, S., Penna, L., Hollis, B. and Thilaganathan, B. (2004) Effect of Parity on SecondTrimester Uterine Artery Doppler Flow Velocity and Waveforms. Ultrasound in Obstetrics and Gynecology, 23, 46-49. http://dx.doi.org/10.1002/uog.908

[18] Harman, C.R. and Baschat, A.A. (2003) Comprehensive Assessment of Fetal Wellbeing: Which Doppler Tests Should Be Performed? Current Opinion in Obstetrics and Gynecology, 15, 147-157. http://dx.doi.org/10.1097/00001703-200304000-00010

[19] Goynumer, G., Yayla, M., Arisoy, R. and Durukan, B. (2009) The Effect of Parity on Midgestational Uterine Artery Doppler Findings in Uncomplicated and Low-Risk Pregnancies. Gynecologic and Obstetric Investigation, 68, 191-195. http://dx.doi.org/10.1159/000232943

[20] Pirhonen, J., Berqersen, T.K., Abdlenoor, M., Dubiel, M. and Gudmundsson, S. (2005) Effect of Maternal Age on Uterine Flow Impedance. Journal of Clinical Ultrasound, 33, 14-17. http://dx.doi.org/10.1002/jcu.20079

[21] Fong, K., Ryan, M.L., Cohen, H., Amankwah, K., Ohlsson, A., Myhr, T., et al. (1996) Doppler Velocimetry of the Fetal Middle Cerebral and Renal Arteries: Interobserver Reliability. Journal of Ultrasound in Medicine, 15, 317-321.

[22] Figueras, F., Fernandez, S., Eixarch, E., Gomez, O., Martinez, J.M., Puerto, B. and Gratacos, E. (2006) Middle Cerebral Artery Pulsatility Index: Reliability at Different Sampling Sites. Ultrasound in Obstetrics and Gynecology, 28, 809-813. http://dx.doi.org/10.1002/uog.2816

[23] Nienhuis, S.J., van Vugt, J.M., Hooqland, H.J., Ruissen, C.J. and de Haan, J. (1988) Interexaminer Variability of Fetal Doppler Velocity Waveforms. Gynecologic and Obstetric Investigation, 25, 152-157. http://dx.doi.org/10.1159/000293764

[24] Gomez, O., Figueras, F., Martinez, J.M., del Rio, M., Palacio, M., Eixarch, E., et al. (2006) Sequential Changes in Uterine Artery Blood Flow Pattern between the First and Second Trimesters of Gestation in Relation to Pregnancy Outcome. Ultrasound in Obstetrics and Gynecology, 28, 802-808. http://dx.doi.org/10.1002/uog.2814

\section{Abbreviations}

HDCP, Hypertensive disorders complicating pregnancy;

IUtA, Left uterine artery;

MCA, Fetal middle cerebral artery;

PSA, Placental bed spiral artery;

PI, Pulsatility index;

RI, Resistance index;

RA, Fetal renal artery;

rUtA, Right uterine artery;

$\mathrm{S} / \mathrm{D}$, Systolic and diastolic ratio;

UmA, Umbilical artery. 